\title{
Aldolase B mutations in Italian families affected by hereditary fructose intolerance
}

G Sebastio, $R$ de Franchis, P Strisciuglio, G Andria, C Dionisi Vici, G Sabetta, R Gatti, N C P Cross, T M Cox

\begin{abstract}
Hereditary fructose intolerance (HFI) is an inborn error of metabolism caused by aldolase $B$ deficiency. The aldolase $B$ gene has been cloned and the following mutations causing HFI have been identified: A149P (a $G \rightarrow C$ transversion in exon 5), A174D (a C $\rightarrow A$ transversion in exon 5), L288 $\triangle \mathrm{C}$ (a base pair deletion in exon 8), and N334K (a G $\rightarrow C$ transversion in exon 9). We have investigated the occurrence of these mutations in 11 Italian patients affected by HFI using PCR and hybridisation to specific oligomers.

We found that four patients were homozygous for the A149P mutation, two patients were homozygous for the A174D mutation, three patients were compound heterozygotes for both the A149P and A174D mutations, one patient was homozygous for the N334K mutation, and one patient did not show any of the reported mutations (HFI diagnosis carried out by aldolase B assay). The L288 $\Delta \mathrm{C}$ mutation has not been found in this survey.
\end{abstract}

Hereditary fructose intolerance (HFI, MIM 22960) is a recessively inherited disease of fructose metabolism caused by aldolase $B$ deficiency. After characterisation of the cDNA, ${ }^{12}$ the study of molecular defects in the

Dipartimento di Pediatria, Universita degli Studi di Napoli, Via S Pansini 5, 80131 Napoli, Italy. G Sebastio, R de Franchis, P Strisciuglio, G Andria

Ospedale Bambin Gesù, Piazza S Onofrio 4, 00165 Roma, Italy.

G Dionisi Vici, G Sabetta

Instituto G Gaslini, Via 5 Magzio, 16148 Genova-Quarto, Italy.

R Gatti

Royal Postgraduate Medical School, Hammersmith Hospital, Du Cane Road, London W12.

N C P Cross, T M Cox

Correspondence to Dr Sebastio.

Received for publication 10 July 1990.

Revised version accepted for publication 20 September 1990. gene for human aldolase B, in relation to HFI, has proved to be of diagnostic value in this disorder. Four mutations of aldolase $B$ have been characterised by Cross $e t a l .^{3-5}$ The first mutation is a $\mathrm{G} \rightarrow \mathrm{C}$ transversion in exon 5 resulting in an amino acid substitution $\mathrm{Ala} \rightarrow$ Pro at position 149 of the protein (A149P). This mutation alters the substrate binding site of aldolase B, dramatically reducing its activity. A further mutation (A174D) is also a transversion $C \rightarrow A$ in exon 5, which results in a $\mathrm{Ala} \rightarrow$ Asp substitution at position 174. A single base pair deletion (L288 $\Delta$ C) has been identified in exon 8 causing a frameshift with a premature stop codon. Finally, a $\mathbf{G} \rightarrow \mathbf{C}$ transversion has been found in exon 9, which results in an Asn $\rightarrow$ Lys substitution at position 334 of the protein (N334K).

We have used the DNA polymerase chain reaction (PCR) ${ }^{6}$ to analyse directly the A149P, A174D, $\mathrm{L} 288 \Delta \mathrm{C}$, and $\mathrm{N} 334 \mathrm{~K}$ mutations in 11 unrelated Italian patients affected by HFI.

\section{Materials and methods}

DNA from peripheral blood of $11 \mathrm{HFI}$ patients from 11 unrelated Italian families was prepared by conventional methods. A $322 \mathrm{bp}$ region of exon 5, containing the mutation site, was amplified using 30 cycles of DNA PCR as follows: $1 \mu \mathrm{g}$ of genomic DNA was mixed with $100 \mu \mathrm{l}$ PCR reaction buffer. Reaction temperatures were: denaturing at $93^{\circ} \mathrm{C}$ for two minutes, annealing at $41^{\circ} \mathrm{C}$ for two minutes, and extension at $71^{\circ} \mathrm{C}$ for two minutes using $\mathrm{Taq}$ DNA polymerase (Cetus, Perkin-Elmer, USA). Sequences for exon 5 were 5'CCC-TTTATTAGAAGCCCATG3' and 5'GGTCCATTTGTAGTTATAGT3'. Amplified DNA was electrophoresed on a $1 \cdot 2 \%$ agarose gel and Southern blotted according to standard procedures. Filters were then hybridised to two different oligomers carrying either the wild (A149) or the mutant (149P) sequence: (A149) 5'AAGTGGCGTGCTGTGCTGA3' and (149P) 5'AAGTGGCGTCCTGTGCTGA3' (fig 1). Filters were then rehybridised to show the presence of the A174D mutation using oligomers identifying either the wild type (A174) or the mutant (A174D) sequence: (A174) 5'TCGCTACGC- 
A149P

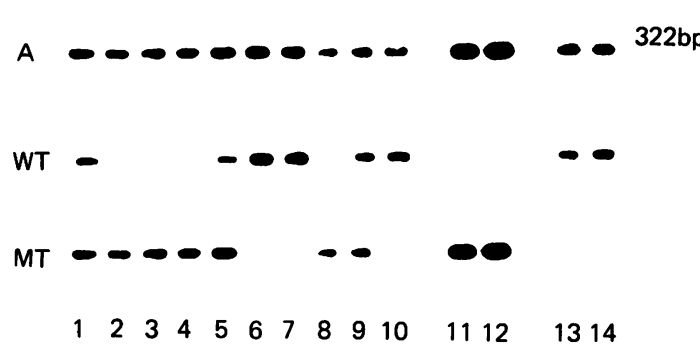

Figure 1 A shows signals after a low stringency washing to check DNA amplification. WT indicates hybridisation to the wild type oligonucleotide. MT indicates hybridisation to the mutant type oligonucleotide. 1 to 10 are patients, 11 and 12 are $A 149 P$ clones used as mutant controls, and 13 and 14 are A149P clones used as wild controls.

AGCATCT3' or (174D) 5'AGATGCTGTCGTAGCGA3' (fig 2). A 279 bp region of exon 8, containing the L288 $\triangle \mathrm{C}$ mutation site, was amplified by PCR using 5'CAGGGTATATAAGGTGGGAC3' and 5'AAGTAGATAAGAGGTGGC3'. The filter obtained after electrophoresis and Southern blotting was hybridised to two other different oligomers recognising either the $L 288 \Delta \mathrm{C}$ mutation or the wild sequence: (L288wt) 5'AATGCTATCAACCTTTG3' and (L288 $\triangle$ C) 5'AATGCTATCAACTTTGC3'. A 207 bp region, including the N334K mutation site, was again amplified by PCR. Sequences were 5'TTCCCATGAGAGGCAGA3' and 5'GCACTGGAGCTAGGCTGG3'.

The filter was then hybridised to wild and mutant oligomers whose sequences are (N334) 5'GCTAACTGCCAGGCGG3' and (334K) 5'GCTAAGTGCCAGGCGG3' (fig 3). Finally, genomic DNA was digested with the restriction enzyme BamHI, electrophoresed on a $\mathbf{0 . 8 \%}$ agarose gel, and blotted. Filters were hybridised to an aldolase B cDNA clone ${ }^{2}$ and

\section{A174D}

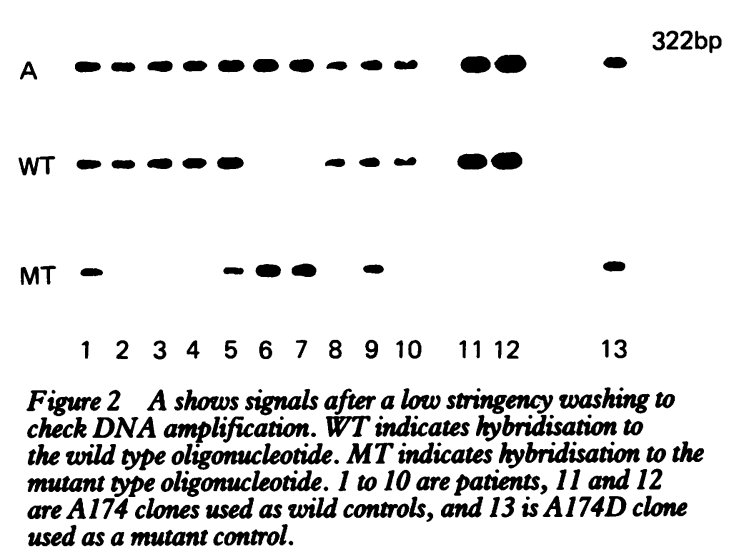

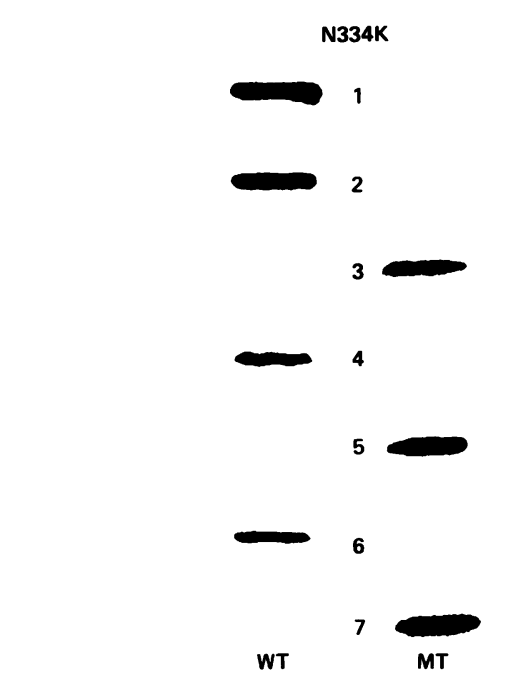

Figure $3 W T$ indicates hybridisation to the wild type oligonucleotide. MT indicates hybridisation to the mutant type oligonucleotide. 1 corresponds to patient 10 in figs 1 and 2,2 is a new HFI patient still under investigation, 3 is a new $H F I$ patient who shows the N334K mutation, 4 and 6 are N334 clones used as wild controls, and 5 and 7 are N334K clones used as mutant controls.

labelled by nick translation with ${ }^{32} \mathbf{P}$ dCTP. This probe hybridises to the entire protein coding region, but does not hybridise to exon 1 and the upstream region.

\section{Results}

The results of DNA molecular analysis, aldolase B enzymatic assay on liver biopsy, and intravenous fructose tolerance test are summarised in the table. No detectable deletions or rearrangements of the aldolase $B$ gene were found in any of our patients on Southern blotting analysis of genomic DNA when digested with BamHI. DNA PCR amplification and hybridisation to oligomers, specific for either the wild

Genotypes of probands.

\begin{tabular}{ccc}
\hline Patients & Mutations & Diagnosis \\
\hline 1 & A149P/A174D & AA \\
2 & A149P/A149P & AA \\
3 & A149P/A149P & AA \\
4 & A149P/A149P & AA \\
5 & A149P/A174D & AA \\
6 & A174D/A174D & FTT \\
7 & A174D/A174D & FTT \& AA \\
8 & A149P/A149P & AA \\
9 & A149P/A174D & AA \\
10 & ? & AA \\
11 & N334k/N334K & $-{ }^{*}$ \\
\hline
\end{tabular}

AA=diagnosis by aldolase $B$ enzymatic assay on liver biopsy. FTT=diagnosis by intravenous fructose tolerance test. *Diagnosis suspected on clinical grounds only. 
sequence or the mutant sequence, gave the following results.

(1) Patients 2, 3, 4, and 8 are homozygous for the A149P mutation. All four were diagnosed on the basis of fructaldolase assays using homogenates of liver biopsy samples. (2) Patients 1,5 , and 9 are compound heterozygotes for the A149P and the A174D mutations and were diagnosed by enzymatic assay. (3) Patients 6 and 7 are homozygous for the A174D mutation. The former was diagnosed on the basis of the intravenous fructose tolerance test and the latter by enzymatic assay. (4) Patient 10, whose diagnosis was carried out by aldolase $B$ enzymatic assay, does not show either the A149P or the A174D mutation. (5) Patient 11, whose diagnosis was made only on the basis of clinical signs, is homozygous for the mutation N334K. None of the patients was found to carry the L288 $\Delta C$ mutation (data not shown).

\section{Discussion}

In this survey of Italian patients, A149P was found to be the most common mutation causing HFI ( $50 \%$ of independent alleles), while the A174D mutation was found in $35 \%$ of the HFI alleles. Three patients were found to be compound heterozygotes for A149P and A174D and their respective parents thus carry one or other of these two prevalent mutant alleles.

The prevalence of the A149P mutation in Italy confirms in part results of a European collaborative study by Cross $e t a l,{ }^{4}$ in which nine independent Italian patients had been already investigated. The frequency of the A149P mutation was found to be greatest in northern European countries and lowest in Italy. On the other hand, A174D is relatively more frequent in HFI patients from southern Europe and appears to be more frequent in Italy. The L288 $\Delta \mathrm{C}$ mutation, found in two Sicilian patients, was not observed in the present study, thus suggesting that it may represent a private mutation or may have been carried by migration from elsewhere in the Mediterranean region. N334K, found in patient number 11 (table), has been previously detected in four Yugoslav patients, in one Austrian, and in one British HFI patient. $^{5}$

The results presented here, together with those of Cross et al, ${ }^{4}$ allow a more accurate estimation of allelic frequencies of A149P, A174D, and 'other' (for example, L288 $\Delta \mathrm{C}$ and $\mathrm{N} 334 \mathrm{~K}$ ) mutations in HFI Italian families: $0.53,0.29$, and 0.18 respectively. In the present study, we have been unable to establish any relationship between clinical presentation and genotype. In fact, both homozygotes for A149P and A174D mutations presented with either severe clinical manifestations in early life or mild symptoms later.

The direct analysis of aldolase B mutations in genomic DNA represents an important advance in the diagnosis of HFI since it is safe, rapid, and definitive. At the time of writing about $90 \%$ of HFI alleles can be identified by this method which, in addition, allows genetic counselling to be offered to heterozygotes.

1 Paolella G, Santamaria R, Izzo P, Costanzo P, Salvatore F. Isolation and nucleotide sequence of a full-length $C D N A$ coding for aldolase B from human liver. Nucleic Acids Res 1984;12: 7401-10.

2 Rottmann WJ, Tolan DR, Penhoet EE. Complete amino acid sequence for human aldolase $B$ derived from $\mathrm{CDNA}$ and genomic clones. Proc Natl Acad Sci USA 1984;81:2738-42.

3 Cross NCP, Tolan DR, Cox TM. Catalytic deficiency of human aldolase $B$ hereditary fructose intolerance caused by a common missense mutation. Cell 1988;53:881-5.

4 Cross NCP, de Franchis R, Sebastio G, et al. Molecular analysis of aldolase B genes in hereditary fructose intolerance. Lancet 1990;335:306-9.

5 Cross NCP, Stojanov LM, Cox TM. A new aldolase B variant, $\mathrm{N} 334 \mathrm{~K}$, is a common cause of hereditary fructose intolerance in Yugoslavia. Nucleic Acids Res 1990;18:1925.

6 Saiki RK, Scharf S, Faloona F, et al. Enzymatic amplification of $\beta$-globin sequences and restriction site analysis for diagnosis of sickle cell anaemia. Science 1985;230:1350-4. 